\title{
Emulsificación de petróleo crudo para su trasporte por oleoductos
}

\author{
Crude Oil Emulsification for Pipeline Transportation
}

\author{
Martínez-Martín Erich \\ Instituto Superior de Tecnologías y Ciencias Aplicadas \\ (InSTEC), Cuba \\ Correo:erich@instec.cu \\ Ramírez-Apodaca Francisco Daniel \\ Instituto Superior de Tecnologías y Ciencias Aplicadas \\ (InSTEC), Cuba \\ Correo:fdramirez@instec.cu
}

\author{
Acosta-Martínez Leonardo \\ Instituto Superior de Tecnologías y Ciencias Aplicadas (InSTEC), \\ Cuba \\ Correo:lacosta@instec.cu
}

Información del artículo: recibido: febrero de 2016, aceptado: marzo de 2016

\section{Resumen}

El agotamiento de las reservas convencionales y el incremento de la demanda de petróleo crudo a nivel mundial originaron constantes innovaciones en los procesos de exploración, producción y trasporte en la industria petrolera. El petróleo pesado y extra pesado, representan una significativa fracción del total de las reservas conocidas. Los oleoductos son sistemas eficientes y económicos para su trasporte, sin embargo, el trasiego de crudo pesado y extra pesado se torna engorroso debido a su elevada viscosidad. Las emulsiones del petróleo con soluciones de tensoactivo resultan eficientes y económicamente viables cuando se elaboran de acuerdo con parámetros que optimizan su uso. La aplicación de esta técnica exige la estabilidad de las emulsiones durante el periodo de bombeo y su completa separación después de transportado el crudo. En el presente trabajo se emulsionaron dos ejemplares de petróleo crudo, utilizando el surfactante no-iónico Tritón X-100 como agente emulsionante. Se analizaron como factores experimentales el contenido de oil, la concentración de tensoactivo, la velocidad de agitación y el tiempo de agitación, asimismo, como variables de respuesta, rectoras de los procesos de trasiego y separación del crudo, la viscosidad y la estabilidad de la emulsión, respectivamente. Los resultados experimentales permiten identificar los factores que mayor influencia presentan sobre las variables de respuesta.

\section{Descriptores:}

- emulsión

- estabilidad

- fluidez

- soluciones de tensoactivo

- viscosidad 


\begin{abstract}
Piping systems (pipelines) are efficient and safe for transporting crude oil. Millions of kilometers of them have been installed and are in operation all over the world. A large amount of crude oil exhibits rheological behavior that impedes the transfer in nature; hence, the need to study techniques for improving the fluidity of the crude. Emulsions of oil with surfactant solutions are efficient and economically viable when processed using parameters that optimize their use. The application of this technique requires stability of the emulsions during the pumping period and their complete separation after transporting the crude. In this paper, two crude oils and the nonionic surfactant Triton X-100 were used. The experimental results allow to identify the factors that have most influence on the pumping and separation process.
\end{abstract}

\section{Introducción}

El agotamiento de las reservas convencionales y el incremento de la demanda de petróleo crudo a nivel mundial han originado constantes innovaciones en los procesos de exploración, producción y trasporte en la industria petrolera (Hart, 2014; Saniere, 2004). El petróleo pesado (densidad menor a $20 \mathrm{API}$ ) según Saniere (2004) y extra pesado, incluyendo el bitumen, representan una significativa fracción del total de las reservas conocidas.

Los oleoductos son los sistemas más eficientes y económicos para el trasporte de petróleo crudo y sus derivados, tanto en tierra como en mar. Sin embargo, el trasiego de crudo pesado y extra pesado se torna engorroso debido a su poca movilidad como consecuencia de su elevada viscosidad.

En la actualidad se desarrollan diferentes estrategias para facilitar el transporte de los crudos pesados por sistemas de tuberías tales como: la dilución con disolventes orgánicos o aceites más ligeros, el uso de mejoradores de flujo, reducción de arrastre mediante aditivos (Calin, 2009) y el calentamiento (Hart, 2014; Palou, 2011).

Otra tecnología que permite mejorar la fluidez del petróleo crudo es la elaboración de una mezcla con fluidos de menor viscosidad y costo, como el agua. La dificultad de esta tecnología radica en que el agua y el petróleo son sustancias químicamente inmiscibles, por lo que la mezcla de ambas origina una emulsión. Si la fase orgánica $(\mathrm{O})$ está dispersa en el agua $(\mathrm{W})$ se denominan emulsiones $\mathrm{O} / \mathrm{W}$, en caso contrario, se denominan emulsiones W/O (Hart, 2014; Palou, 2011). Las emulsiones por lo general son sistemas inestables, cuya estabilidad depende de agentes emulsionantes o tensoactivos (Salager, 2002). La presencia de tensoactivos en la interfase agua/petróleo permite controlar los fenómenos involucrados con la tensión superficial durante la etapa de drenaje/floculación (Salager, 1991), cuya escala de tiempo puede variar en función de la concentración del tensoactivo. Los surfactantes no-iónicos son los agentes emulsionantes más utilizados en la industria petrolera por ser relativamente baratos, no variar sus efectos producto a la salinidad y ser biodegradables (Palou, 2011; Kumar 2014; Fernández, 2004).

El trasporte de petróleo crudo emulsionado por oleoductos se divide en tres etapas fundamentales: emulsificación del crudo, trasporte del crudo emulsionado y desemulsificación para usos industriales posteriores, donde el contenido de agua es inferior a $2 \%$ (Hart, 2014; Saniere, 2004; Palou, 2011). Las principales variables involucradas en estos procesos son: la viscosidad de la mezcla y la estabilidad de la emulsión.

El objetivo de la presente investigación consiste en identificar los principales factores del proceso de emulsificación que influyen en la viscosidad y la estabilidad de las emulsiones. Se estudian como factores experimentales: contenido de oil, concentración de tensoactivo, velocidad de agitación y tiempo de agitación.

\section{Materiales y métodos}

En el trabajo experimental se utilizaron dos ejemplares de petróleo crudo, adquiridos en la Empresa Comercializadora de Combustibles Matanzas, perteneciente a la compañía petrolera “Cuba Petróleo" (Cupet). En la tabla 1 se muestran algunas propiedades de ambos fluidos, relevantes para el trabajo experimental desarrollado. El crudo A clasifica como petróleo crudo medio poco viscoso, mientras el crudo B resulta pesado de elevada viscosidad (Saniere, 2004; Vahid, 2012).

Tabla 1. Principales propiedades de los crudos medio (A) y pesado (B)

\begin{tabular}{|c|c|c|c|}
\hline Propiedades & UM & Crudo A & Crudo B \\
\hline Densidad $25^{\circ} \mathrm{C}$ & API & 20.4 & 11.0 \\
\hline Viscosidad $25^{\circ} \mathrm{C}$ & cSt & 13.3 & 344.1 \\
\hline Agua por destilación & $\%$ & 0.7 & 1.3 \\
\hline
\end{tabular}

Para emulsionar los crudos con agua se elaboraron, de antemano, soluciones con el agente tensoactivo no-ióni- 
co Tritón X-100, con fórmula química $\mathrm{C}_{33} \mathrm{H}_{60} \mathrm{O}_{10}$ (Ghannam, 2007; Abdurahman y Yunus, 2013; Ashrafizadeh, 2010; Liyana, 2014; Yousef, 2004; Abdurahman, 2012). En todos los casos, el crudo se añadió lentamente a la solución (Liyana, 2014; Shadi, 2010), mientras se mezclaba utilizando un agitador estándar modelo MR-25 con hélice de tres palas (Abdurahman y Yunus, 2013) y velocidad variable de hasta $2500 \mathrm{rpm}$. Las muestras se elaboraron con un volumen total de $200 \mathrm{~mL}$ en un recipiente de $500 \mathrm{~mL}$ para evitar el derrame de fluido durante la agitación.

El diseño de experimento se desarrolló a partir del software StatGraphics XV - CENTURION. Se analizaron los factores experimentales: contenido de oil (crudo) (50\% - 80\%) (Ashrafizadeh, 2010; Liyana, 2014), concentración de tensoactivo $(0.3 \%-2.5 \%$ ) (Abdurahman y Yunus, 2013; Abdurahman, 2012), velocidad de agitación (1000 rpm - 2500 rpm) (Abdurahman y Yunus, 2013; Abdurahman, 2012), y tiempo de agitación (60 s - 180 s). Las variables de respuesta que se estudiaron: viscosidad (cSt), por su influencia en el proceso de trasiego de crudo; y estabilidad de emulsión (\%) como variable rectora del proceso de desemulsificación.

El trabajo experimental se organizó en dos bloques, utilizando ambos crudos. En el primero se elaboraron
10 muestras con el crudo A, analizando todos los factores experimentales y variables de respuesta anteriormente mencionados. La tabla 2 muestra las combinaciones de factores experimentales propuestas por el software y los valores de viscosidad y estabilidad de la emulsión obtenidos para ellos.

En el segundo bloque se elaboraron solo 5 muestras, utilizando el crudo B. En este caso, se estableció un tiempo de agitación fijo de 120 segundos, en aras de obtener mayor claridad en los resultados experimentales. Esto permitió identificar los factores que mayor influencia presentan sobre la viscosidad y la estabilidad de la emulsión como variables respuesta (tabla 3).

Para la determinación de la viscosidad se utilizó un viscosímetro de Engler modelo B3-246, cuyo principio de funcionamiento se basa en el tiempo de vaciado del fluido en cuestión, respecto al mismo volumen de agua a igual presión y temperatura (Martínez, 2015).

La estabilidad de la emulsión se obtuvo a partir de la ecuación 1 (Abdurahman y Yunus, 2013; Liyana, 2014; Abdurahman, 2012; Rasha, 2014). Para ello, se utilizaron recipientes de volumen total $50 \mathrm{~mL}$, donde las muestras permanecieron en reposo hasta su total separación (Alabdulmohsen, 2015; Hartland, 2007). El valor de estabilidad seleccionado para el análisis experimen-

Tabla 2. Diseño de experimento para el crudo A

\begin{tabular}{|c|c|c|c|c|c|c|}
\hline Muestra & $\begin{array}{c}\text { Contenido de oil } \\
(\%)\end{array}$ & $\begin{array}{l}\text { Concentración } \\
\text { tensoactivo (\%) }\end{array}$ & $\begin{array}{c}\text { Velocidad agitación } \\
(\mathrm{rpm})\end{array}$ & $\begin{array}{c}\text { Tiempo } \\
\text { agitación (s) }\end{array}$ & $\begin{array}{l}\text { Viscosidad } \\
\text { (cSt) }\end{array}$ & $\begin{array}{c}\text { Estabilidad } \\
\text { emulsión (\%) }\end{array}$ \\
\hline 1 & 50 & 2.5 & 1000 & 180 & 8.03 & 61 \\
\hline 2 & 80 & 2.5 & 2500 & 180 & 10.49 & 100 \\
\hline 3 & 80 & 0.3 & 2500 & 60 & 10.35 & 80 \\
\hline 4 & 65 & 1.4 & 1750 & 120 & 8.76 & 88 \\
\hline 5 & 65 & 1.4 & 1750 & 120 & 8.41 & 87 \\
\hline 6 & 50 & 2.5 & 2500 & 60 & 7.60 & 73 \\
\hline 7 & 80 & 2.5 & 1000 & 60 & 8.79 & 75 \\
\hline 8 & 50 & 0.3 & 1000 & 60 & 7.97 & 29 \\
\hline 9 & 80 & 0.3 & 1000 & 180 & 9.70 & 84 \\
\hline 10 & 50 & 0.3 & 2500 & 180 & 7.68 & 36 \\
\hline
\end{tabular}

Tabla 3. Diseño de experimento para el crudo B

\begin{tabular}{cccccc}
\hline Muestra & $\begin{array}{c}\text { Contenido de oil } \\
(\%)\end{array}$ & $\begin{array}{c}\text { Concentración tensoactivo } \\
(\%)\end{array}$ & $\begin{array}{c}\text { Velocidad agitación } \\
(\mathrm{rpm})\end{array}$ & $\begin{array}{c}\text { Viscosidad (cSt) } \\
\text { Estabilidad emulsión } \\
(\%)\end{array}$ & $\begin{array}{c}\text { (\%) } \\
1\end{array}$ \\
\hline 10 & 2.5 & 2500 & 23.16 & 100 \\
2 & 80 & 0.3 & 1000 & 22.51 & 60 \\
3 & 50 & 0.3 & 2500 & 8.49 & 46 \\
4 & 50 & 2.5 & 1000 & 7.56 & 50 \\
5 & 65 & 1.4 & 1750 & 7.36 & 48 \\
\hline
\end{tabular}


tal (tablas 2 y 3), corresponde al estado de la emulsión después de trascurrida una hora de elaboración.

\section{Resultados y discusión}

El trasporte de petróleo crudo emulsionado se divide en tres etapas fundamentales: emulsificación del crudo, trasporte del crudo emulsionado y desemulsificación para usos industriales posteriores. Las principales variables involucradas en estos procesos son la viscosidad de la mezcla y la estabilidad de la emulsión. Los factores experimentales que mayor influencia ejercen sobre estas son: contenido de oil, concentración de tensoactivo, velocidad de agitación y tiempo de agitación (Ghannam, 2007; Abdurahman y Yunus, 2013; Ashrafizadeh, 2010; Liyana, 2014; Shadi, 2010; Rasha, 2014; Alabdulmohsen, 2015; Tarragól, 2012; Nehal y Nael, 1999; Abdel, 2012). A continuación se identifican los principales factores experimentales que obedecen las variables de respuesta mencionadas anteriormente, variando el rango y las combinaciones de estos.

\section{Efectos sobre la viscosidad}

Se analizaron diferentes factores experimentales utilizando ambos crudos (A y B). La figura 1 muestra la influencia de cada factor sobre la viscosidad para el crudo Aa y para el crudo Bb, a partir del diagrama de Pareto. Este permite identificar en qué medida los componentes rigen el comportamiento de una variable. En ambos casos, el factor dominante resultó el contenido de oil, donde el resultado es consecuencia de la inversión de la emulsión (de $\mathrm{O} / \mathrm{W}$ a W/O) que ocurre cuando el crudo se convierte en la fase continua. Generalmente dicho fenómeno tiene lugar para un contenido de oil superior a 70\% (Abdurahman y Yunus, 2013; Ashrafizadeh, 2010; Nehal y Nael, 1999), dependiendo del tipo de crudo.

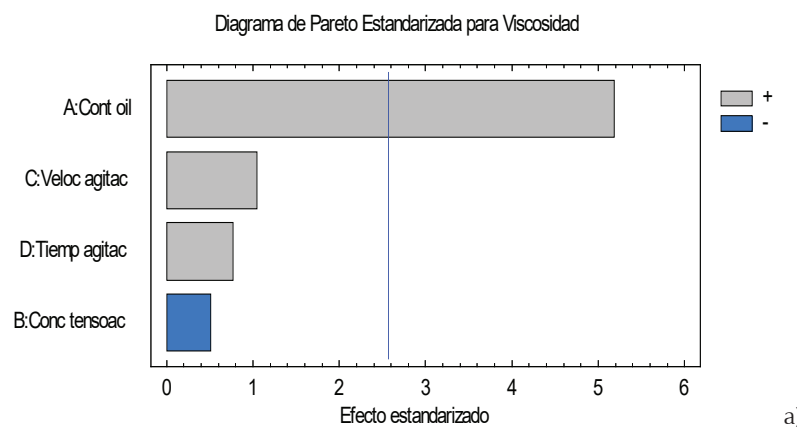

El segundo factor más influyente fue la velocidad de agitación, en menor medida para el crudo B. Al aumentar la velocidad de agitación se incrementan los esfuerzos cortantes (cizallamiento) sobre las gotas de ambas fases, lo que provoca la disminución de su tamaño. Este efecto trae consigo el incremento de la superficie de contacto entre el crudo y las paredes del ducto ofreciendo mayor resistencia al movimiento, por lo que la mezcla se torna más viscosa.

La figura 2 representa la dependencia de la viscosidad respecto a la velocidad de agitación y el contenido de oil. Tanto para el crudo Aa como para el Bb, es posible mantener constante la viscosidad de la mezcla aumentando el contenido de oil y disminuyendo la velocidad de agitación, y viceversa. Por ejemplo, el crudo A, presenta igual viscosidad de $8.56 \mathrm{cSt}$ para un contenido de oil de $65 \%$ y velocidad de agitación de 1000 rpm que para un contenido de oil de $59 \%$ y velocidad de agitación de $2500 \mathrm{rpm}$. Este aspecto ofrece un margen para la elaboración de la emulsión, permitiendo ajustar los parámetros de trabajo a las condiciones del sistema de bombeo.

Como muestra la figura 1 el factor que menor influencia ejerce sobre la viscosidad es la concentración de tensoactivo. A diferencia del resto de los factores, con el aumento de esta la viscosidad disminuye. Tal efecto se debe a la reducción de la tensión interfacial entre el fluido y el ducto que produce el agente tensoactivo facilitando su movilidad. En el caso del crudo B (figura 1, b) es prácticamente despreciable la influencia de la concentración de tensoactivo sobre la viscosidad de la mezcla debido a la elevada viscosidad del crudo, incluso emulsionado. En la figura 3 se aprecia el comportamiento de la viscosidad con las variaciones del contenido de oil y la concentración de tensoactivo. Para ambos crudos el valor más elevado de viscosidad se alcanza para el mayor contenido de oil y la menor concentración de tensoactivo.

Figura 1. Diagrama de Pareto para los factores que influyen en la viscosidad, a) crudo A, b) crudo B 

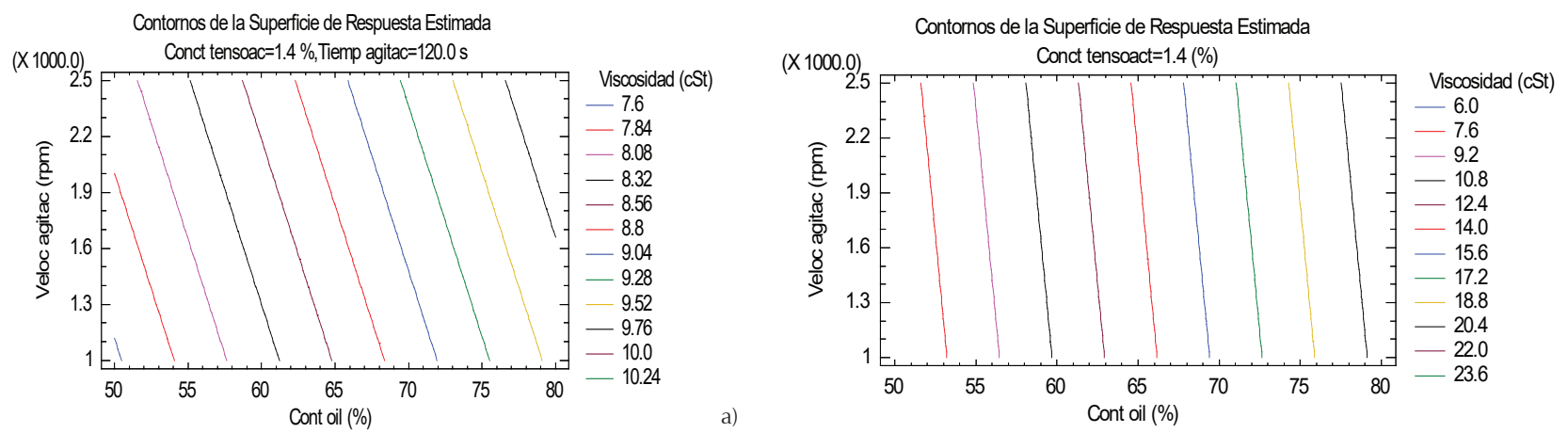

b)

Figura 2. Influencia de la velocidad de agitación y el contenido de oil sobre la viscosidad, a) crudo A, b) crudo B
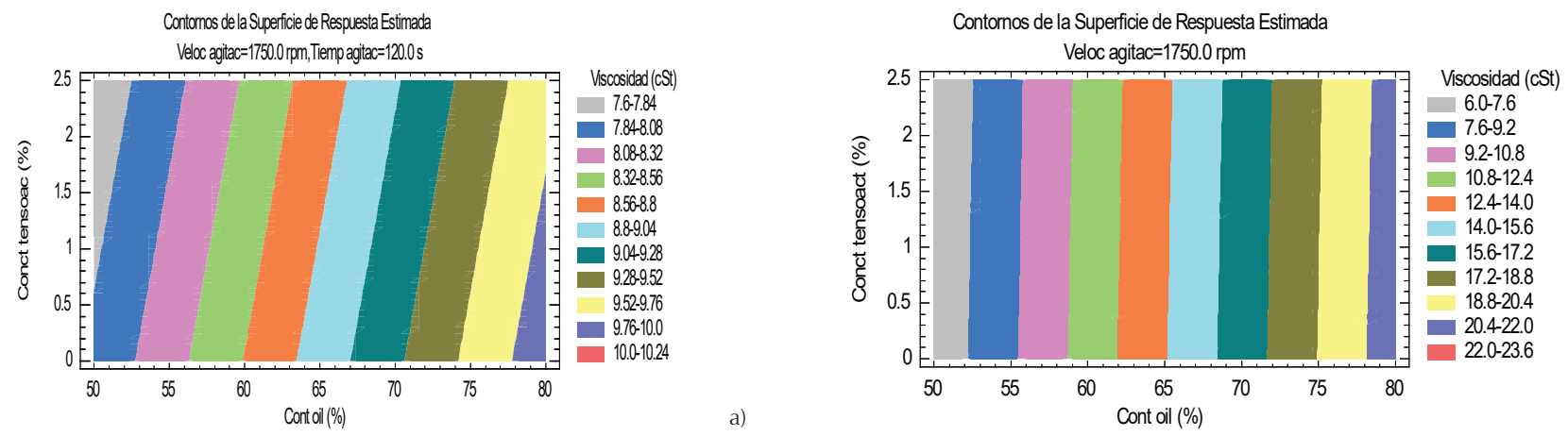

b)

Figura 3. Influencia de la concentración de tensoactivo y el contenido de oil sobre la viscosidad, a) crudo A, b) crudo B

El tiempo de agitación y la velocidad de agitación se encuentran estrechamente vinculados, por ser ambos factores propios del proceso de mezclado. La figura 4 representa la relación inversa entre ambos elementos. Es posible obtener la misma viscosidad de 8.8 cSt para una velocidad de agitación de 2400 rpm y 60 s de agitación que para una velocidad de $1300 \mathrm{rpm}$ y tiempo de agitación de 180 s. La combinación de ambos factores puede variar de acuerdo con las condiciones mecánicas de la instalación y el caudal de crudo trasegado. Para el caso de la variable estabilidad de la emulsión se observa un comportamiento similar.

\section{Estabilidad de emulsiones}

La figura 5 representa la influencia de los factores experimentales sobre la estabilidad de las emulsiones, para el crudo Aa y el crudo Bb. El contenido de oil, en ambos casos se mantiene como factor predominante. Esto se debe al efecto que provocan las fuerzas viscosas asociadas al crudo sobre las gotas de agua, actuando como inhibidor del proceso de drenaje-floculación. El segundo factor más influyente sobre la estabilidad de las emulsiones, es la concentración de tensoactivo. La acción estabilizadora de este último permite la unión en- tre ambas fases actuando como enlace, lo que retarda la coalescencia de las gotas de agua. La velocidad de agitación y el tiempo de mezclado siguen comportamientos semejantes al expuesto en la figura 4.

La figura 6 muestra la influencia de la concentración de tensoactivo y el contenido de oil sobre la estabilidad de la emulsión manteniendo una velocidad de agitación constante de 1750 rpm y tiempo de agitación igual a 120 s. En ambos casos (a y b) el máximo valor de estabilidad se alcanza para el mayor contenido de oil y la mayor concentración de tensoactivo. La emulsión del crudo B presenta $83 \%$ de estabilidad, después de una hora en reposo, tanto para un contenido de oil de $80 \%$ y concentración de tensoactivo de $0.3 \%$ como para un contenido de oil de $50 \%$ y una concentración de tensoactivo de $1.9 \%$. Este resultado ofrece un rango de variación en los parámetros de trabajo que permite seleccionar las condiciones de emulsificación adecuadas al proceso, utilizando el menor volumen de tensoactivo posible. Por ejemplo, para un contenido de oil de $70 \%$ (punto de inversión de las fases) la emulsión del crudo B presenta la misma estabilidad de $83 \%$ utilizando solo una concentración de tensoactivo de $0.8 \%$.

La figura 7 representa el comportamiento de las muestras 2, 4 y 8 (tabla 2) del crudo A. La muestra 2 se 


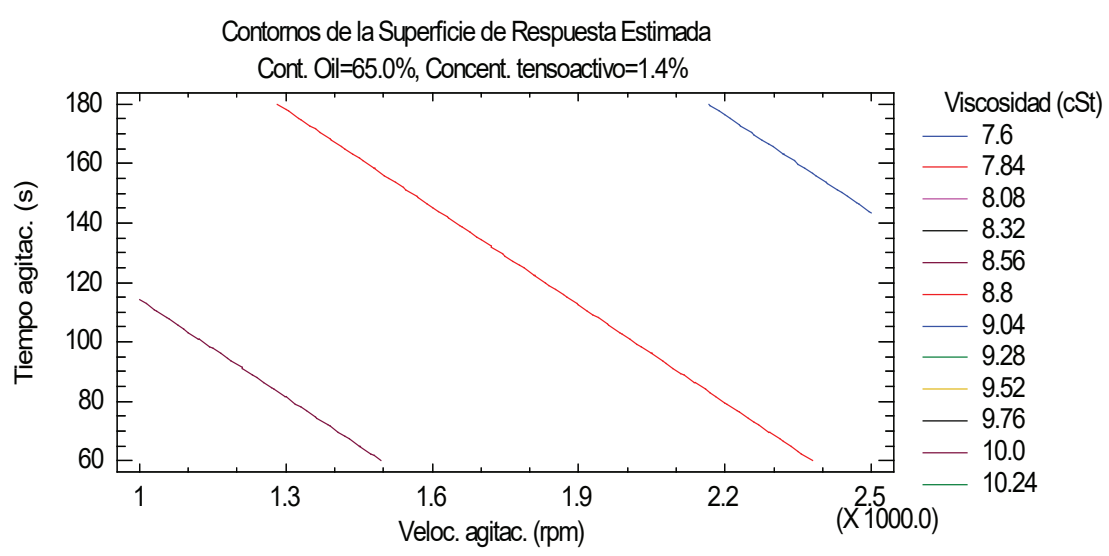

Figura 4. Relación entre la velocidad de agitación y el tiempo de agitación para el crudo A
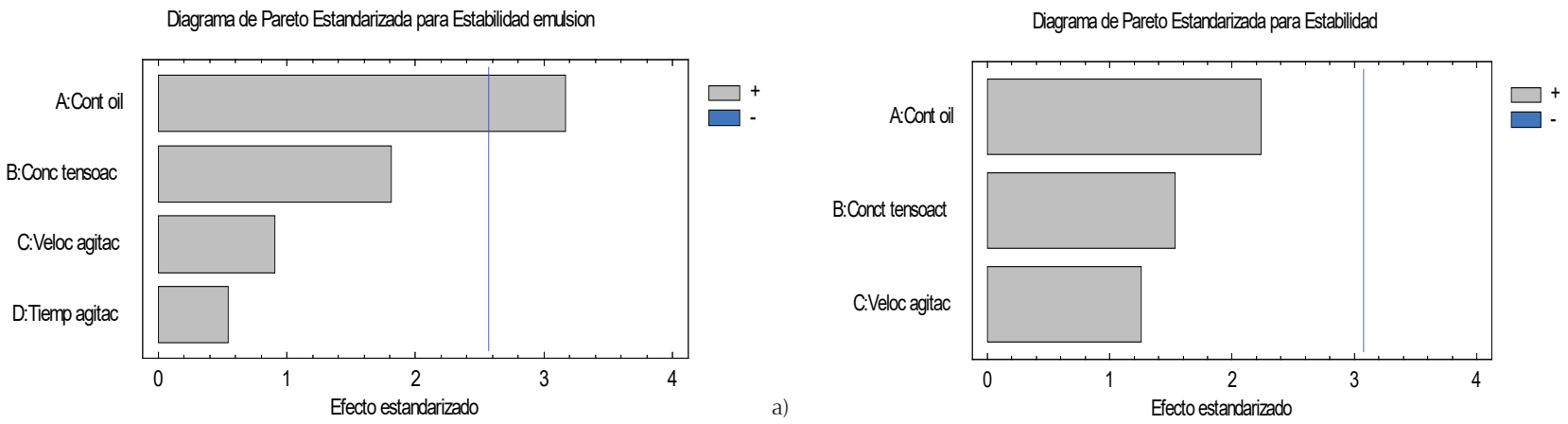

Figura 5. Diagrama de Pareto para los factores que influyen en la estabilidad de la emulsión, a) crudo A, b) crudo B
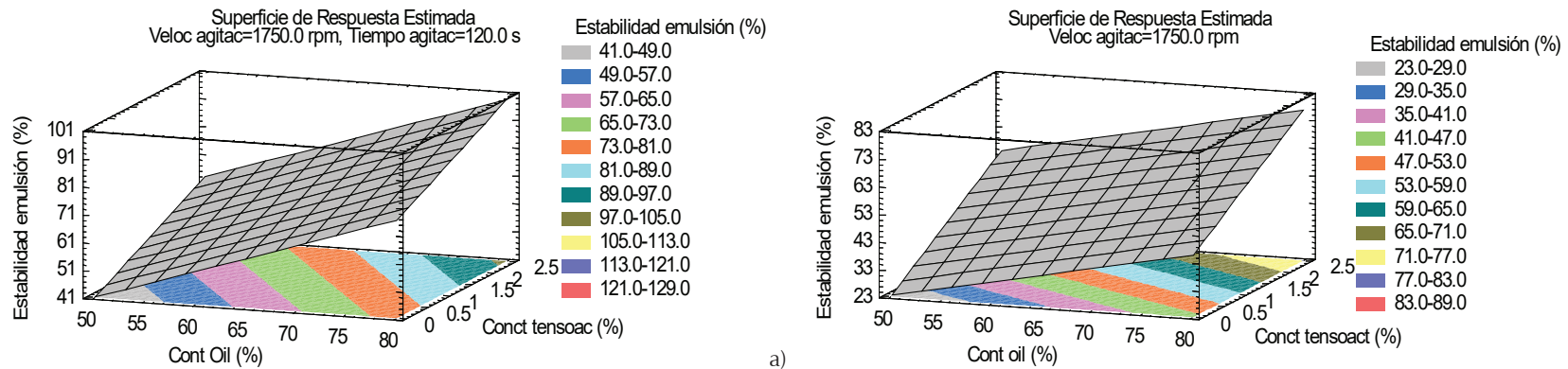

Figura 6. Influencia de la concentración de tensoactivo y el contenido de oil en la estabilidad de la emulsión, a) crudo A, b) crudo B 


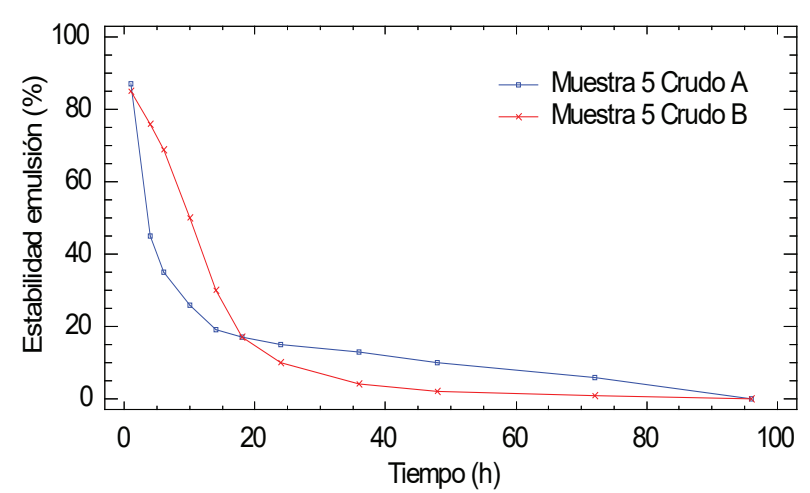

Figura 7. Estabilidad de emulsión del crudo A

elaboró utilizando la combinación de factores que genera mayor estabilidad, la muestra 4 con valores medios y la muestra 8 con la combinación menos estable. Trascurridas 10 horas la muestra 8 se separó $90 \%$, la muestra 4 , $80 \%$, y la muestra 2 se mantuvo $100 \%$ estable.

Como tendencia general las emulsiones presentan mayor velocidad de separación cuanto más contenido de agua posean. Este fenómeno se debe a que con el aumento de la fase continua existe mayor contacto entre las gotas de agua, facilitando la unión de estas en una nueva de mayor tamaño y peso (coalescencia) (Langevin et al., 2004). Esta última se arrastra por la fuerza de gravedad hacia el fondo del recipiente, venciendo los esfuerzos viscosos del crudo. Ello explica por qué la mayor separación tiene lugar en las primeras horas del proceso (aproximadamente 90\%) y luego ocurre un retardo en la separación de $10 \%$ de agua restante en la mezcla.

En la figura 8 se observan los procesos de separación que tienen lugar para las muestras número 5 del crudo $\mathrm{A}$ (tabla 2) y del crudo B (tabla 3). Ambas emulsiones se elaboraron utilizando igual combinación de factores experimentales y condiciones de separación. Aunque las curvas presentan comportamientos similares, es apreciable la estabilidad de la emulsión $B$ en la etapa inicial respecto a la muestra A. Sin embargo, después de trascurridas 17 horas, ambas muestras exhiben igual estabilidad de $19 \%$. A partir de este momento la muestra B aumenta su velocidad de separación en relación con la muestra A. Esta disparidad tiene sus orígenes en los diferentes fenómenos físico-químicos que tienen lugar en la mezcla como consecuencias de la composición y propiedades termofísicas de ambos crudos.

\section{Conclusiones}

Se elaboraron 15 muestras de emulsiones utilizando dos tipos de crudo y el surfactante no-iónico Tritón

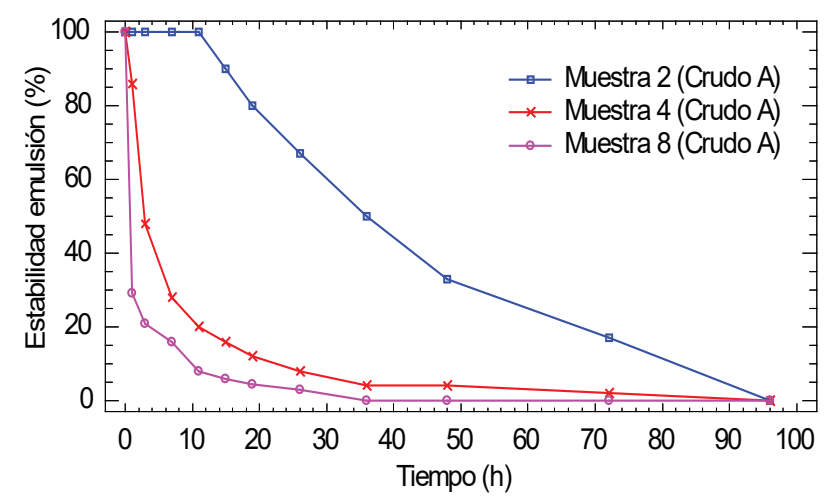

Figura 8. Comparación entre la estabilidad de emulsión del crudo A y el crudo B

X-100 como agente estabilizador de la emulsión. Se analizaron los factores experimentales: contenido de oil, concentración de tensoactivo, velocidad de agitación y tiempo de agitación y las variables de respuesta: viscosidad y estabilidad de la emulsión.

La viscosidad de las emulsiones para ambos crudos estudiados es directamente proporcional al contenido de oil, la velocidad de agitación y el tiempo de agitación; y disminuye con el aumento de la concentración de tensoactivo, donde el contenido de oil es el factor de mayor influencia. En el caso del crudo B los efectos de la concentración de tensoactivo sobre la viscosidad son insignificantes.

Los factores que mayor influencia ejercen sobre la estabilidad de las emulsiones son el contenido de oil y la concentración de tensoactivo, para ambos tipos de crudo. Los resultados ofrecen un rango de variación en los parámetros de trabajo que permiten seleccionar las condiciones de emulsificación adecuadas al proceso.

Como tendencia general las emulsiones presentan mayor velocidad de separación cuanto más contenido de agua posean, la mayor separación tiene lugar en las primeras horas del proceso y luego ocurre un retardo en la separación de $10 \%$ de agua restante en la mezcla. El crudo B es más estable en la primera etapa del proceso respecto al crudo $\mathrm{A}$, sin embargo, después de trascurridas 17 horas ambas muestras exhiben igual estabilidad.

\section{Referencias}

Abdel-Raouf M.E.S. Factors affecting the stability of crude oil emulsions, in crude oil emulsions- composition stability and characterization, Abdul-Raouf P.M.E.S., editor, 2012, intech: egypt [en línea]. Disponible en: http://www.intechopen.com/books/ crude-oil-emulsions-composition-stability-and-characteriza- 
tion/factors-affecting-the-stability-of-crude-oil-emulsions. Isbn: 978-953-51-0220-5.

Abdurahman N.H., N.H.A. y Yunus M. Formulation and evaluation of water-continuous emulsion of heavy crude oil prepared for pipeline transportation. International Journal of Engineering Science and Innovative Technology (IJESIT), volumen 2 (número 5), 2013: 170-179.

Abdurahman N.H. Pipeline transportation of viscous crudes as concentrated oil-in-water emulsions. Journal of Petroleum Science and Engineering, volumen 90, (número 6), 2012 [en línea]. Disponible en: http://dx.doi.org/10.1016/j.petrol.2012.04.025.

Ashrafizadeh S.N., M.K. Emulsification of heavy crude oil in water for pipeline transportation. Journal of Petroleum Science And Engineering, volumen 71 (números 205-211), 2010 [en línea]. Disponible en: www.elsevier.com/locate/petrol.

Alabdulmohsen Z. Experimental study of crude oil emulsion stability by surfactants and nanoparticles, Missouri University of Science and Technology, 2015.

Calin A. The influence of drag-reducing additives on crude oil emulsions in pipeline flow. U.P.B. Sci. Bull., volumen 71 (número 3), 2007: 197-204.

Fernández A. J.L.S. Surfactantes noiónicos, Cuaderno firp núm. 303pp, U.D.L. Andes editor, 2004, Laboratorio Firp, Escuela de Ingeniería Química, pp. 1-28 [en línea]. Disponible en: www.firp.ula.ve.

Ghannam M.T. Water-in-crude oil emulsion stability investigation. Petroleum Science and Technology, volumen 23 (números 649-667), 2007 [en línea]. Disponible en: http://dx.doi. org/10.1081/lft-200033001. Issn: 1091-6466.

Hart A. A review of technologies for transporting heavy crude oil and bitumen via pipelines. J Petrol Explor Prod Technol., volumen 4 (número 1), 2014: 327-336.

Hartland A.B.S. Study of demulsification of water-in-crude oil emulsion. Journal Of Dispersion Science and Technology, volumen 14 (número 5), 2007: 541-557 [en línea]. Disponible en: http://dx.doi.org/10.1080/01932699308943426.

Kumar R. Studies on the effect of surfactants on rheology of synthetic crude. Journal Of Petroleum Science Research, volumen 3, (número 2), 2014, pp. 90-99 [en línea]. Disponible en: www.jpsr.org.

Langevin D.S.P., Hénaut I., Argillier J.F. Crude oil emulsion properties and their application to heavy oil transportation. Oil \& Gas Science and Technology, volumen 59 (número 5), 2004: 511-521.

Liyana M.S, A.H.N. Stabilization and characterization of heavy crude oil in-water $(\mathrm{o} / \mathrm{w})$ emulsions. International Journal of Re- search in Engineering and Technology, volumen 3 (número 2), 2014: 489-496 [en línea]. Disponible en: www.ijret.org.

Martínez-Martín E. M.D.L.V.P.D., Olmo-Velázquez A. Empleo de emulsiones con soluciones de tensoactivo para el transporte de sustancias de elevada viscosidad. Revista cubana de ingeniería, volumen VI (número 1), 2015: 51-56.

Nehal S.A., A.M.N., Nael N.Z. Formation of fluid heavy oil-inwater emulsions for pipeline transportation. Fuel, volumen 78 (número 593-600), 1999.

Palou R.M. Transportation of heavy and extra-heavy crude oil by pipeline: a review. Journal of Petroleum Science And Engineering, volumen 75 (número 1), 2011: 274-282.

Rasha-Mohammed A., A.H.N. Kinetic stability and rheology of water-in-crude oil emulsion stabilized by cocamide at different water volume fractions. International Journal of Chemical Engineering and Applications, volumen 5 (número 2), 2014: 204-209.

Salager J.L. Fenómenos interfaciales en dispersiones polifásicas y medios porosos. Cuaderno firp núm. 303pp, U.D.L. Andes editor, 2002, Laboratorio Firp, Escuela de Ingeniería Química, pp. 1-38 [en línea]. Disponible en: www.firp.ula.ve.

Salager J.L. Uso de los surfactantes en la industria petrolera. Cuaderno firp 350a, U.D.L. Andes editor, 1991, Laboratorio Firp, Escuela de Ingeniería Química, pp. 1-17 [en línea]. Disponible en: www.firp.ula.ve.

Shadi W.H. y Nabil-Esmail M.T.G. Heavy crude oil viscosity reduction and rheology for pipeline transportation. Fuel, volumen 89 (números 1095-1100), 2010 [en línea]. Disponible en: www.elsevier.com/locate/fuel.

Saniere A. I.H.a.J.F.A. Pipeline transportation of heavy oils, a strategic, economic and technological challenge. Oil \& Gas Science And Technology, volumen 59 (número 5), 2004: 455-466.

Tarragói H.L.R. Estabilidad de emulsiones acuosas de petróleo crudo pesado con agente emulsionante de productos de pirólisis. Tecnología Química, volumen 33 (número 3), 2012: 253-263.

Vahid-Hoshyargar A.M. Prediction of flow behavior of crude oilin-water emulsion through the pipe by using rheological properties. Oriental Journal of Chemistry, volumen 28 (número 1), 2012: 109-113 [en línea]. Disponible en: www.orientjchem.org.

Yousef Al-Roomia R.G. Elgibalyb A., Elkamela A. Use of a novel surfactant for improving the transportability/ transportation of heavy/viscous crude oils. Journal of Petroleum Science and Engineering, volumen 42 (números 235-243), 2004 [en línea]. Disponible en: www.sciencedirect.com. 


\section{Este artículo se cita:}

\section{Citación estilo Chicago}

Martínez-Martín, Erich, Francisco Daniel Ramírez-Apodaca, Leonardo Acosta-Martínez. Emulsificación de petróleo crudo para su trasporte por oleoductos. Ingeniería Investigación y Tecnología, XVII, 03 (2016): 395-403.

\section{Citación estilo ISO 690}

Martínez-Martín E., Ramírez-Apodaca F.D., Acosta-Martínez L. Emulsificación de petróleo crudo para su trasporte por oleoductos. Ingeniería Investigación y Tecnología, volumen XVII (número 3), julio-septiembre 2016: 395-403.

\section{Semblanzas de los autores}

Erich Martínez-Martín. Ingeniero en tecnologías nucleares y energéticas, pertenece al Instituto Superior de Tecnologías y Ciencias Aplicadas de la Facultad de Ciencias y Tecnologías Nucleares.

Leonardo Acosta-Martínez. Estudiante de ingeniería en tecnologías nucleares y energéticas en el Instituto Superior de Tecnologías y Ciencias Aplicadas de la Facultad de Ciencias y Tecnologías Nucleares.

Francisco Daniel-Ramírez. Estudiante de ingeniería en tecnologías nucleares y energéticas, Apodaca, Instituto Superior de Tecnologías y Ciencias Aplicadas, Facultad de Ciencias y Tecnologías Nucleares.

Erich Martínez-Martín. Ingeniero en tecnologías nucleares y energéticas, pertenece al Instituto Superior de Tecnologías y Ciencias Aplicadas de la Facultad de Ciencias y Tecnologías Nucleares. Ha publicado artículos en: Revista Cubana de Ingeniería, Revista de Ingeniería Mecánica, Revista de Ingeniería Energética y Revista Chilena de Ingeniería.

Leonardo Acosta-Martínez. Estudiante de ingeniería en tecnologías nucleares y energéticas en el Instituto Superior de Tecnologías y Ciencias Aplicadas de la Facultad de Ciencias y Tecnologías Nucleares. Ha publicado artículos en: Revista Cubana de Ingeniería.

Francisco Daniel-Ramírez. Estudiante de ingeniería en tecnologías nucleares y energéticas, Apodaca, Instituto Superior de Tecnologías y Ciencias Aplicadas, Facultad de Ciencias y Tecnologías Nucleares. Ha publicado artículos en: Revista Cubana de Ingeniería. 